\title{
Development and Evaluation of an Optical Sensing System for Detection of Herbicide Spray Droplets
}

\author{
Yanbo Huang1*, Wei Ma², Daniel Fisher ${ }^{3}$ \\ ${ }^{1}$ USDA-ARS Crop Production Systems Research Unit, Stoneville, Mississippi, USA \\ ${ }^{2}$ Institute of Urban Agriculture, Chinese Academy of Agricultural Sciences, Chengdu, China \\ ${ }^{3}$ USDA-ARS Sustainable Water Management Research Unit, Stoneville, Mississippi, USA \\ Email: *yanbo.huang@usda.gov
}

How to cite this paper: Huang, Y.B., Ma, W. and Fisher, D. (2021) Development and Evaluation of an Optical Sensing System for Detection of Herbicide Spray Droplets. Advances in Internet of Things, 11, 1-9. https://doi.org/10.4236/ait.2021.111001

Received: December 3, 2020

Accepted: January 18, 2021

Published: January 21, 2021

Copyright ( 2021 by author(s) and Scientific Research Publishing Inc. This work is licensed under the Creative Commons Attribution International License (CC BY 4.0).

http://creativecommons.org/licenses/by/4.0/

\section{(c) (i) Open Access}

\begin{abstract}
Real time monitoring of herbicide spray droplet drift is important for crop production management and environmental protection. Existing spray droplet drift detection methods, such as water-sensitive paper and tracers of fluorescence and Rubidium chloride, are time-consuming and laborious, and the accuracies are not high in general. Also, the tracer methods indirectly quantify the spray deposition from the concentration of the tracer and may change the drift characteristics of the sprayed herbicides. In this study, a new optical sensor system was developed to directly detect the spray droplets without the need to add any tracer in the spray liquid. The system was prototyped using a single broadband programmable LED light source and a near infrared sensor containing 6 broadband spectral detectors at $610,680,730,760,810$, and 860 $\mathrm{nm}$ to build a detection system for monitoring and analysis of herbicide spray droplet drift. A rotatory structure driven by a stepper motor in the system was created to shift the droplet capture line going under the optical sensor to measure and collect the spectral signals that reflect spray drift droplets along the line. The system prototype was tested for detection of small (Very Fine and Fine), medium (Medium), and large (Coarse) droplets within the droplet classifications of the American Society of Agricultural and Biological Engineers. Laboratory testing results indicated that the system could detect the droplets of different sizes and determine the droplet positions on the droplet capture line with $100 \%$ accuracy at the wavelength of $610 \mathrm{~nm}$ selected from the 6 bands to detect the droplets.
\end{abstract}

\section{Keywords}

Near Infrared (NIR) Sensor, Spray Drift, Droplet Detection, Plant Protection 


\section{Introduction}

Spray application of herbicides is an important means to control weeds in crop fields. In general, the purpose of using pesticides in crop production is to help ensure the yields of crops and to preserve the environment surrounding the crop fields at the same time. Specifically, the impact of herbicides on crop production is huge and in the United States, more than $95 \%$ of crop lands for maize, cotton, soybean and sugarbeet are treated with herbicides for weed control [1]. However, because of some uncontrollable factors, especially weather factors, herbicides can protect targeted crops but their off-target drift may also be negative to susceptible crops and the environment [2] [3]. Crops can suffer unpredictable damage when applied herbicides drift off-target close to sensitive crops [4] [5] [6]. Although the spray drift is usually very small relatively to the total amount of spray, it is still of great concern to producers. Smith et al. [7] indicated that even ultra-low herbicide drift will still have a clear negative response to the yield of cotton. The assessment of the risk of herbicide drift has been widely studied [8]. In the United States, aerial and ground applications of herbicides have been widely adopted, and problems of off-target drift are serious concerns. The spray drift is influenced by many dynamic factors, such as spray droplets generated by different system configurations and under different weather conditions, during the spray operation [6] [9] [10].

To assess the impact of spray drift, an effective method of spray droplet detection is critical [11]. Existing spray droplet detection methods include using mylar sheet and water-sensitive paper along with imaging techniques for tracer intensity, such as fluorescence [12], Rubidium chloride ( $\mathrm{RbCl}$ ) [13], and droplet sizes [14]. Spray tracers are typically mixed in the spray tank with the herbicide to measure the concentration of the tracer and indirectly obtain the amount of herbicide spray deposition and drift. However, the added tracer may change the true herbicide spray characteristics, so the insight of the herbicide spray and drift under certain conditions may be misleading. Water-sensitive paper provides a rough estimate of spray droplet sizes relying on discoloration of water on the specially made paper. These methods are laborious and time consuming.

This study was undertaken to develop an optical sensing system to directly measure spray distribution with droplet sizes without need of adding any tracer in the spray liquid, which has not done before by anybody else. The system is built with inexpensive, open-source electronic hardware and software and nearinfrared (NIR) spectroscopy.

\section{Materials and Methods}

\subsection{System Prototype}

The spray droplet detection system consists of a microcontroller-based circuit designed to detect and measure the distribution of herbicide (chemical) droplets deposited on a capture line string. The system includes a programmable microcontroller, multiband NIR sensor, stepper motor, and droplet capture line string, 
and is configured as a portable, battery-powered monitoring system. The main components are described in the following section, with a schematic diagram of the system prototype shown in Figure 1 and a listing of the components described in Table 1.

\subsection{Programmable Microcontroller}

The Feather M0 Adalogger microcontroller development platform (Adafruit Industries, New York, NY, USA; https://adafruit.com) integrates a programmable microcontroller, microSD card circuit, and auxiliary voltage regulation and passive electronic components into a single circuit board. The resulting circuit board features convenient access to the microcontroller's input/output pins and features, regulated $3.3 \mathrm{~V}$ output power supply, USB connection for uploading of program instructions, and microSD card data storage.

The microcontroller consists of an ATSAMD21G18 ARM Cortex M0 32-bit processor, which operates at a clock speed of $48 \mathrm{MHz}$ and a $3.3 \mathrm{~V}$ logic level. Built-in memory includes 256 Kbytes of FLASH for user-written program storage and 32 Kbytes of RAM for program run-time operations. Access is provided to 20 input/output pins, which support serial, I2C, and SPI digital communications

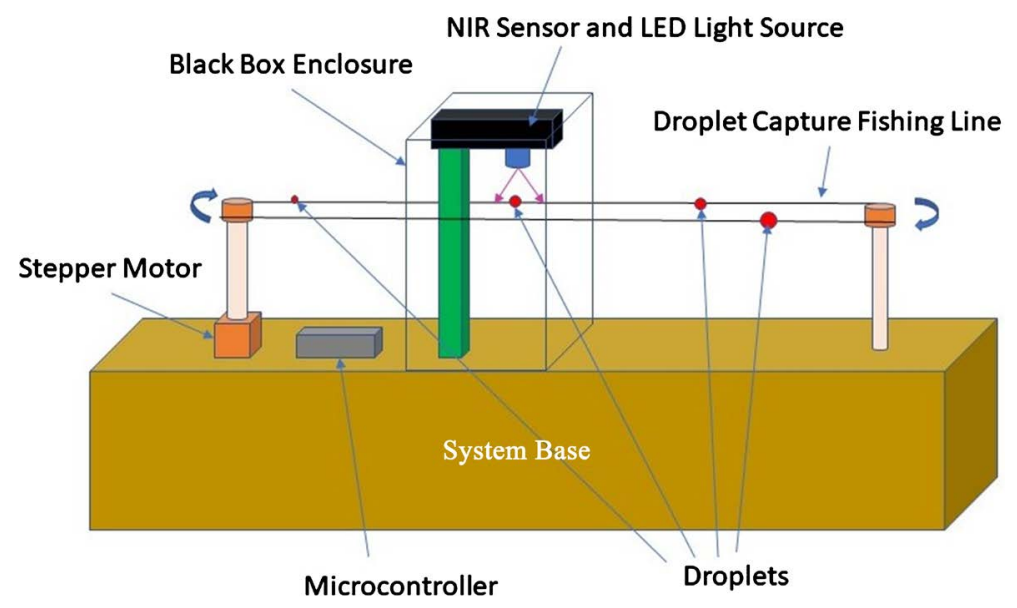

Figure 1. Schematic diagram of the optical spray droplet detection system prototype.

Table 1. List of materials and approximate costs for the droplet detection system.

\begin{tabular}{cccc}
\hline \multirow{2}{*}{ Main components } & \multicolumn{3}{c}{ Materials and costs list } \\
\cline { 2 - 4 } & Part number & Supplier & Cost (USD) \\
\hline Microcontroller board & Feather M0 Adalogger & Adafruit Industries & 20 \\
NIR sensor board & AS7263 & Sparkfun Electronics & 26 \\
Stepper motor & NEMA-17 & Adafruit Industries & 14 \\
Motor driver & NBF-32002 & Adafruit Industries & 5 \\
Rechargeable battery & 5 V LiPoly, 5000 mAh & Adafruit Industries & 10 \\
12 V battery & & & 10 \\
Miscellaneous & & & 6 \\
& & & Total: 91 \\
\hline
\end{tabular}


protocols, provide multiple 12-bit analog-to-digital converters, and enable multiple PWM (pulse-width modulation) outputs.

The inclusion of a microSD card circuit enables convenient and reliable user-data storage. Voltage regulation and logic-level shifting components meet voltage level and timing requirements to facilitate proper functioning of data read and write operations to ensure accurate data transfer.

The microcontroller is programmed using the open-source Arduino Integrated Development Environment, or IDE,

(https://www.arduino.cc/en/Main/Software), a software utility downloaded to and installed on a personal computer. The IDE enables the user to write programs that control the operation of the microcontroller, compile and error-check the program, and upload the resulting code to the microcontroller. The IDE programming language is based on the $\mathrm{C}++$ programming language and supports the inclusion of external program libraries. Libraries contain programming routines that provide certain features or control specific peripheral devices and sensors and can be included to more rapidly and easily incorporate advanced features into a user's program.

\subsection{Near Infrared Sensor}

The AS7263 Near-Infrared (NIR) Spectral Sensor breakout board (Sparkfun Electronics, Niwot, CO, USA; https://sparkfun.com) was selected as the sensing element of the droplet detection system. The breakout board consists of a multiband NIR sensor, as well as auxiliary electronic components, mounted on a single circuit board. The NIR sensor, the AS7263 Spectral Sensing Engine (ams AG, Premstaetten, Austria; https://ams.com) is a six-channel spectrometer that measures reflectance in near infrared light wavelengths and consists of independent optical filters that measure $20-\mathrm{nm}$ wavebands centered at six wavelengths (Table 2).

The NIR sensor communicates with the microcontroller via the I2C (Inter Integrated Circuit) digital communications protocol.

The NIR sensor was coupled with a light source and shroud to provide and maintain a stable, consistent source of light for illuminating and detecting droplets. An adjustable LED light (Ber-lite31-31-52, Bausch \& Lomb Inc., NY, USA) was evaluated at various color temperatures and light intensities.

Table 2. Wavelengths of the NIR sensor.

\begin{tabular}{cc}
\hline Channel & Wavelength (nm) \\
\hline R & 610 \\
S & 680 \\
T & 730 \\
U & 760 \\
V & 810 \\
W & 860 \\
\hline
\end{tabular}




\subsection{Stepper Motor}

A stepper motor is used to move the droplet capture line in precise increments and position the line in view of the NIR sensor. The NEMA-17 stepper motor (Adafruit Industries) is a 4-wire bipolar stepper motor that operates from a $12-\mathrm{V}$ supply and is controlled with a resolution of 200 steps/revolution (1.8 $8^{\circ}$ per step). A TB6612 DC/Stepper Motor Driver Breakout Board (Adafruit Industries) interfaces the stepper motor with the microcontroller and is used to control the operation of the stepper motor. The motor driver board contains two full H-bridges, allowing bi-directional control of two DC motors or one 4-wire stepper motor.

\subsection{Droplet Capture Line}

The droplet capture line string consists of commonly available nylon fishing line. To evaluate the potential impact of the type and color of the fishing lines used, lines with four different colors (red, moss green, green, and clear) and three different diameters $(0.25,0.38$, and $0.46 \mathrm{~mm})$ were purchased for testing.

\subsection{Assembled System Prototype}

The prototype system depicted in Figure 1 was assembled for testing and evaluation. A circuit board was fabricated for mounting the electronic components and consisted of a protoboard and male and female headers. Female headers were arranged and soldered to the protoboard to mount the microcontroller and motor driver boards, which mated via male headers soldered to the two boards. Male headers were soldered to the protoboard to connect the NIR sensor via a short cable soldered to the sensor board. A schematic of the electronic circuit is shown in Figure 2.

The microcontroller was programmed to manage all sensor, stepper motor, and data storage functions. When electrical power was supplied to the system, the microcontroller program began execution by first running a setup routine, which configured input/output pins, initialized the NIR sensor and motor driver,

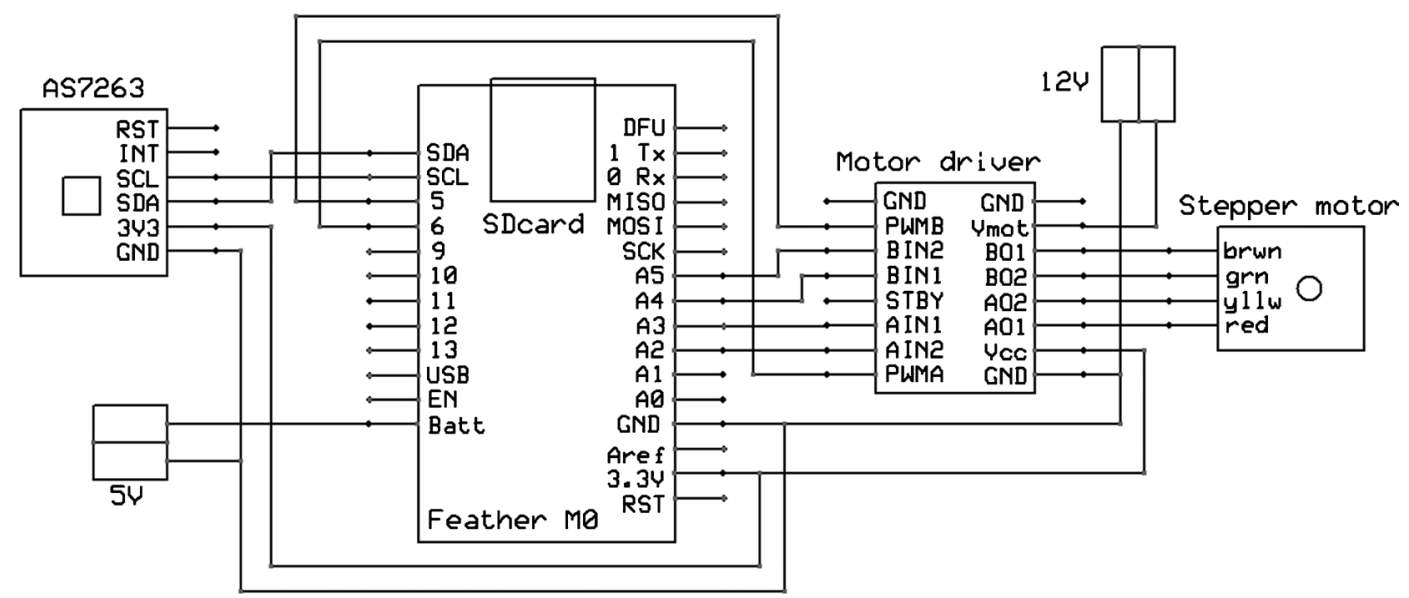

Figure 2. Electronic schematic of the system prototype. 
and set up the microSD card for data transfer.

The sensor measurement and data-collection process then began. The stepper motor was instructed to rotate 20 steps, and the droplet capture line advanced approximately $10 \mathrm{~mm}$ and stopped. The microcontroller sent instructions via I2C to the NIR sensor, which returned measurements from each of the six NIR waveband channels. The six NIR measurements were then stored to the microSD card. Program execution returned to the beginning of the sensor-measurement routine, and the process was repeated. The microcontroller program is open-source and is freely available by contacting the authors.

\subsection{System Testing}

The droplet detection system was tested in the laboratory using an Eppendorf pipette (Eppendorf AG, Hamburg, Germany) of $0.1 \mu \mathrm{l}-2.5 \mu \mathrm{l}$ volume to generate droplets of varying volumes with approximate diameters of 300,400 , and 500 $\mu \mathrm{m}$ corresponding with centers of the ASABE (American Society of Agricultural and Biological Engineers) droplet VMD (Volume Medium Diameter) categories of medium $(236-340 \mu \mathrm{m})$, coarse $(341-403 \mu \mathrm{m})$, and very coarse (404 - 502 $\mu \mathrm{m})[15]$, respectively. Testing started by examining the effect of droplet capture line colors to determine the color of line used in the system, and then testing the system performance for detection of droplets of various sizes.

\section{Results}

For system testing, the LED light was set to 99 for the Color Rendering Index (CRI) and 3250 for the Kelvin Color Temperature. A shroud was constructed from black cardboard to completely cover the NIR sensor and LED light to block external light interference and actively illuminate the droplet samples with only the LED light.

\subsection{Effect of Droplet Capture Line Colors}

LED light is a mixture of many colors for white light. The LED light is reflected by the droplet deposited and adhered on the different colors of the droplet capture fishing line after spraying, so the light intensity of specific wavelengths will be affected. In the study, a test was conducted to investigate whether the effect of line color on the spectrum was significant. For this test, fishing lines of 4 different colors and 3 different cross-sectional diameters as described above were tested. The test indicated that the red fishing line is the most sensitive in $610 \mathrm{~nm}$ $(\mathrm{R})$, then $680 \mathrm{~nm}(\mathrm{~S})$, wavebands, and others insignificant regardless of diameters (Figure 3). Following testing, red fishing line (ZS1412, Red Lightnin, Zebco, Inc., OK, USA) was used in the system.

\subsection{Droplet Detection}

For calibration of droplet size, 9 droplets of three different particle sizes were placed on the fishing line and the position coordinates of the droplets on the 
fishing line were prerecorded. Five droplets were in the ranges of ASABE Very Fine (VF) $(61-105 \mu \mathrm{m})$ and Fine (F) $(106-235 \mu \mathrm{m})$ droplets. In the test the five droplets were generated by $1 / 3$ and $1 / 2$ of the $300 \mu \mathrm{m}$ droplets generated by the pipette and categorized as small size droplets in the analysis. Two droplets belonged to ASABE Medium (M), which were categorized as medium size droplets in the analysis. Two droplets belonged to ASABE Coarse (C), which were categorized as large size droplets. In detection of droplets, all 9 droplets were accurately detected. The success rate of the detection was 100\%. Droplet selection was randomized, and the test was repeated three times. The coordinates of the droplets were acquired by the sensor and the coordinate values set in advance were well matched. Data were measured in the dynamic environment of the moving fishing line driven by the stepper motor. Figure 4 shows the detection of the 9 droplets at the 6 wavelengths of the NIR sensor, especially at $610 \mathrm{~nm}$ and $680 \mathrm{~nm}$ with significant responses.

Each curve in Figure 4 is the average of 11 replicated tests. With repeated testing, multiple depositions of the herbicide would contaminate the fishing line,

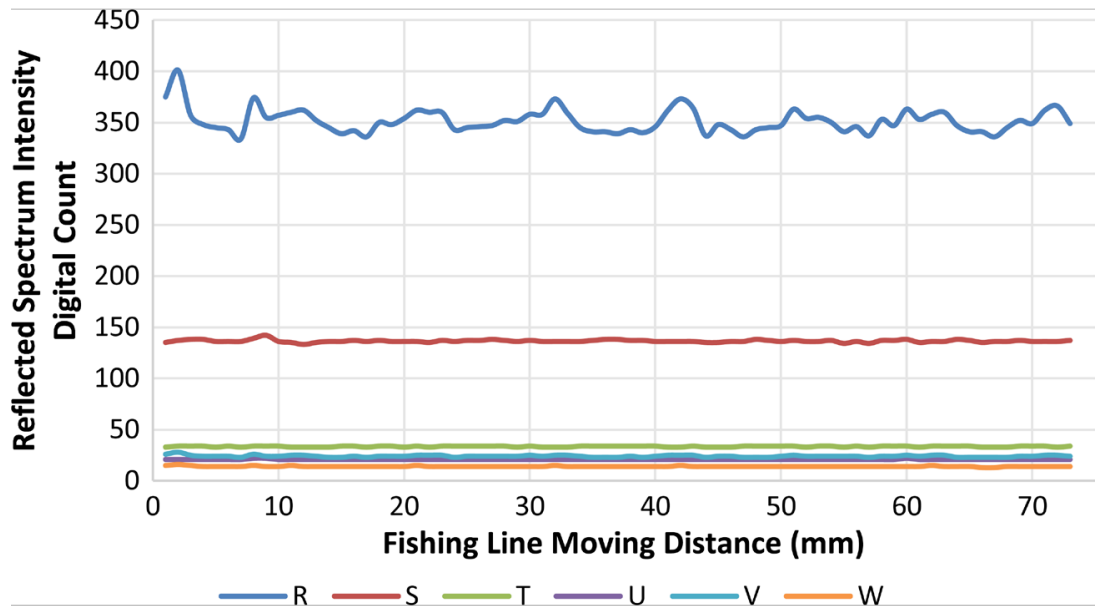

Figure 3. Effect of fishing line colors to 6 wavelengths of the NIR sensor.

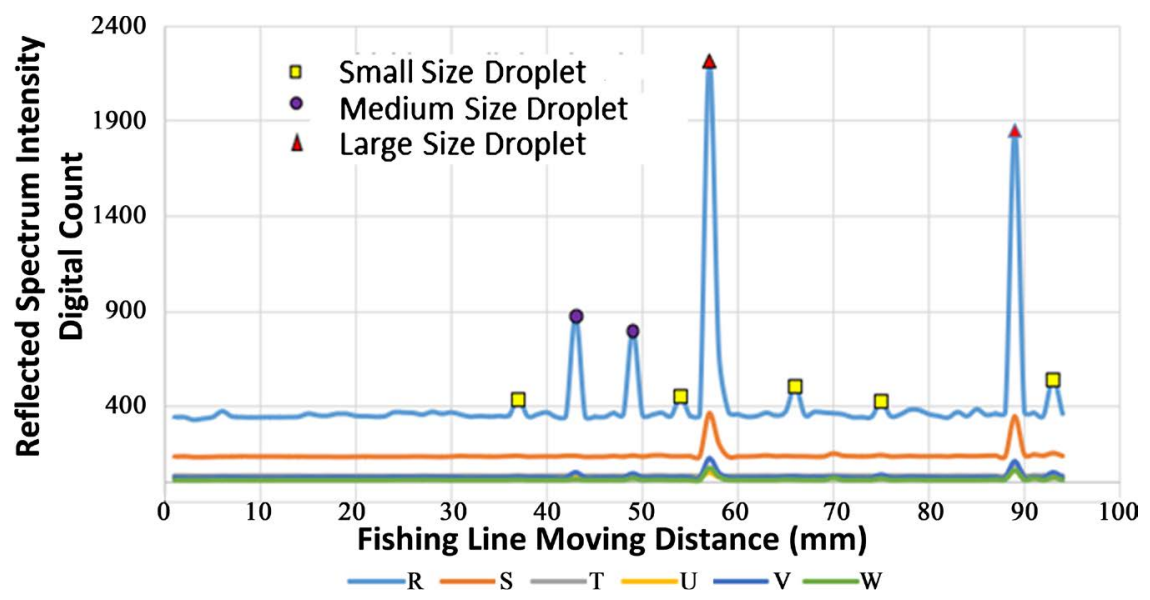

Figure 4. Detection of 9 droplets distributed over the fishing line at 6 wavelengths of the NIR sensor. 
so, a new line (1300 $\mathrm{mm}$ long) was replaced each time during the test. In the range of VF, F, M and C droplets, the standard deviations were small for the $\mathrm{M}$ and $\mathrm{C}$ droplets, which indicate that the relatively large droplets had larger areas of the droplet reflection surface to offer more reliable measurements.

\section{Conclusion}

In this study, an optical spray droplet detection system prototype was developed without the need of adding any tracer in the herbicide spray tank mix. The system prototype was developed with inexpensive open-source electronics and software and tested with droplets of different sizes within ASABE categories. The system reliably detected varied sized droplets, especially $\mathrm{M}$ and $\mathrm{C}$ droplets with large enough areas of the droplet reflection surface on the droplet capture fishing line. With the results of this study, the next step will be to build and test a multiple droplet capture line system prototype to detect herbicide drift in the field.

\section{Conflicts of Interest}

The authors declare no conflicts of interest regarding the publication of this paper.

\section{References}

[1] Gianessi, L.P. (2005) Economic and Herbicide Use Impacts of Glyphosate-Resistant Crops. Pest Management Science, 61, 241-245. https://doi.org/10.1002/ps.1013

[2] Teske, M.E., Bird, S.L., Esterly, D.M., Curbishley, T.M., Ray, S.L. and Perry, S.G. (2002) AgDRIFT $^{\circledR}$ : A Model for Estimating Near-Field Spray Drift from Aerial Applications. Environmental Toxicology and Chemistry, 21, 659-671.

https://doi.org/10.1002/etc.5620210327

[3] Teske, M.E., Thistle, H.W. and Ice, G.G. (2003) Technical Advances in Modeling Aerially Applied Sprays. Transactions of the ASAE, 46, 985-996. https://doi.org/10.13031/2013.13955

[4] Smith, D.B., Bode, L.E. and Gerard, P.D. (2000) Predicting Ground Boom Spray Drift. Transactions of the ASAE, 43, 547-553. https://doi.org/10.13031/2013.2734

[5] Huang, Y., Hoffmann, W.C., Lan, Y. and Fritz, B.K. (2009) Development of a Spray System on an Unmanned Aerial Vehicle Platform. Applied Engineering in Agriculture, 25, 803-809. https://doi.org/10.13031/2013.29229

[6] Huang, Y., Zhan, W., Fritz, B.K., Thomson, S.J. and Fang, A. (2010) Analysis of Impact of Various Factors on Downwind Deposition Using a Simulation Method. Journal of ASTM International, 7, 1-11. https://doi.org/10.1520/JAI102771

[7] Smith, H.C., Ferrell, J.A., Webster, T.M. and Fernandez, J.V. (2017) Cotton Response to Simulated Auxin Herbicide Drift Using Standard and Ultra-Low Carrier Volumes. Weed Technology, 31, 1-9.

[8] Brain, R.A., Perine, J., Cooke, C., Butler, E.C., Harrington, P., Lane, A., Sullivan, C. and Ledson, M. (2017) Evaluating the Effects of Herbicide Drift on Non-Target Terrestrial Plants: A Case Study with Mesotrione. Environmental Toxicology and Chemistry, 36, 2465-2475. https://doi.org/10.1002/etc.3786

[9] Fritz, B.K. (2006) Meteorological Effects on Deposition and Drift of Aerially Ap- 
plied Sprays. Transactions of the ASABE, 49, 1295-1301.

https://doi.org/10.13031/2013.22038

[10] Fritz, B.K., Bagley, B., Hoffmann, W.C. and Lan, Y. (2008) Spray Spectrum Modifications through Changes in Airspeed to Minimize Drift. NAAA/ASABE Paper No. AA08-002, National Agricultural Aviation Association, Washington DC.

[11] Carlsen, S.C., Spliid, N.H. and Svensmark, B. (2006) Drift of 10 Herbicides after Tractor Spray Application. 2. Primary Drift (Droplet Drift). Chemosphere, 64, 778-786. https://doi.org/10.1016/j.chemosphere.2005.10.060

[12] Wen, Y., Zhang, R., Chen, L., Huang, Y., Yi, T. and Xu, G. (2019) A New Spray Deposition Pattern Measurement System Based on Spectral Analysis of a Fluorescent Tracer. Computers and Electronics in Agriculture, 160, 14-22.

https://doi.org/10.1016/j.compag.2019.03.008

[13] Huang, Y., Ouellet-Plamondon, C.M., Thomson, S.J. and Reddy, K.N. (2017) Characterizing Downwind Deposition of the Off-Target Drift from Aerially Applied Glyphosate Using $\mathrm{RbCl}$ as Tracer. International Journal of Agricultural and Biological Engineering, 10, 31-36.

[14] Huang, Y. and Thomson, S.J. (2020) Field Evaluation of a Tri-Set Spray Nozzle for Aerial Application and Discussion on Release of Biological Control Agents. International Journal of Precision Agricultural Aviation, 3, 40-47. https://doi.org/10.33440/j.ijpaa.20200302.75

[15] ASABE (2009) S572.1: Spray Nozzle Classification by Droplet Spectra. ASABE, St. Joseph. 